International Journal of Fatigue

Elsevier Editorial system(tm) for

Manuscript Draft

Manuscript Number:

Title: Effects of Laser Shock Peening on the Microstructure and Fatigue Crack Propagation Behaviour of Thin AA2024 Specimens

Article Type: Original Research Paper

Keywords: Laser shock peening; AA2024; EBSD; fatigue crack Propagation; residual stress

Corresponding Author: Dr. Nikolai Kashaev, Dr.

Corresponding Author's Institution: Helmholtz-Zentrum Geesthacht, Institute of Materials Research, Materials Mechanics

First Author: Nikolai Kashaev, Dr.

Order of Authors: Nikolai Kashaev, Dr.; Volker Ventzke, Dr.; Manfred Horstmann; Sergey Chupakhin; Stefan Riekehr; Rielson Falck; Emad Maawad, Dr.; Peter Staron, Dr.; Norbert Schell, Dr.; Norbert Huber, Prof.

Manuscript Region of Origin: Europe 


\section{Cover Letter}

Title: Effects of Laser Shock Peening on the Microstructure and Fatigue Crack Propagation Behaviour of Thin AA2024 Specimens

Authors: N. Kashaev, V. Ventzke, M. Horstmann, S. Chupakhin, S. Riekehr, R. Falck, E. Maawad, P. Staron, N. Schell, N.Huber International Journal of Fatigue

Geesthacht, November, the $27^{\text {th }} 2016$

Dear Editor,

The enclosed manuscript contains our research work performed in Helmholtz-Zentrum Geesthacht, Institute of Materials Research, Materials Mechanics. The goal of our study was to understand the effects of laser shock peening (LSP) residual stresses on the fatigue crack propagation (FCP) behaviour of the commonly used aircraft aluminium alloy AA2024 under T351 heat treatment condition. LSP introduced high and deep compressive stresses without severe surface damages as well as deformations in the microstructure of thin AA2024 specimens. It is an effective process for improving the resistance against FCP and can therefore be applied to prevent cracks and decelerate the FCP. An original methodology to consider LSP-induced residual stresses on the FCP behaviour was proposed. To our knowledge, there are only few studies that investigate fatigue crack retardation mechanisms acting on materials with LSP residual stresses. This was the basic motivation for the preparation of the presented manuscript.

I suggest the following reviewers for our manuscript:

1. Prof. Xiang Zhang

Professor of Structural Integrity

Coventry University

Department of Mechanical and Automotive Engineering

Coventry, UK

E-Mail: xiang.zhang@coventry.ac.uk

2. Professor Paulo M.S.T. de Castro

Universidade do Porto

Faculdade de Engenharia

Rua Dr. Roberto Frias

4200-465 Porto, Portugal

E-mail: ptcastro@fe.up.pt 
3. Prof. Dr. José L. Ocaña

Centro Láser UPM

Campus Sur UPM. Edificio "La Arboleda".

Ctra. de Valencia, km 7,3.

28031 Madrid, Spain

Depto. Física Aplicada e Ingeniería de Materiales

E.T.S. de Ingenieros Industriales

C/ José Gutiérrez Abascal, 2

28006 Madrid, Spain

Tel.: Office: +34 913363099, +34913365535

Mobile: +34680218670

E-mail: jlocana@etsii.upm.es

I declare, that the submission is original and is not being submitted for publication elsewhere.

With best regards,

Nikolai Kashaev

(on behalf of all co-authors) 

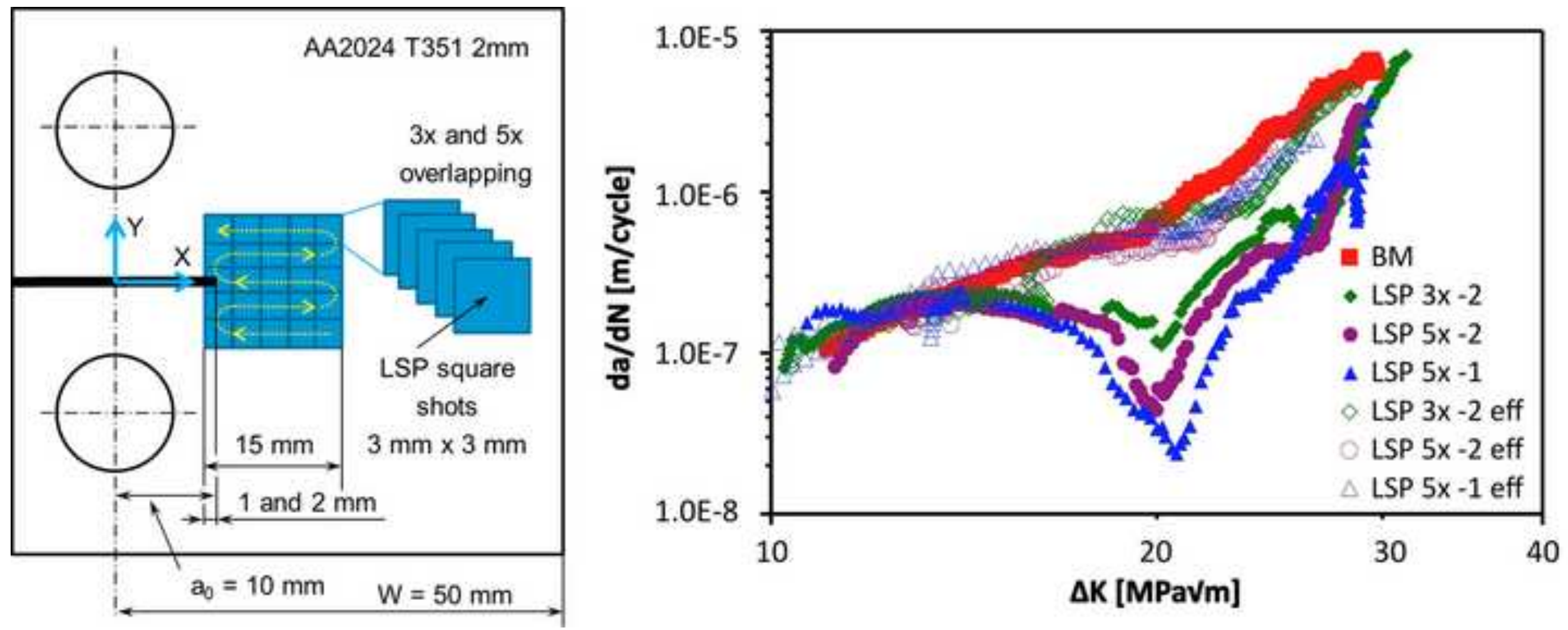


\section{Highlights}

- Deep compressive residual stresses introduced without severe surface damages

- Significant retardation of FCP due to compressive residual stresses observed

- LSP compressive residual stresses caused crack closure effect

- The crack closure effect increased minimum load level and reduced load amplitude

- A methodology to consider the LSP residual stresses for the FCP behaviour proposed 


\title{
Effects of Laser Shock Peening on the Microstructure and Fatigue Crack Propagation Behaviour of Thin AA2024 Specimens
}

\author{
Nikolai Kashaev, Volker Ventzke, Manfred Horstmann, Sergey Chupakhin, Stefan \\ Riekehr, Rielson Falck, Emad Maawad, Peter Staron, Norbert Schell, Norbert Huber \\ Institute of Materials Research, Helmholtz-Zentrum Geesthacht, \\ Max-Planck-Str. 1, 21502 Geesthacht, Germany \\ nikolai.kashaev@hzg.de
}

\begin{abstract}
Highlights
- Deep compressive residual stresses introduced without severe surface damages

- Significant retardation of FCP due to compressive residual stresses observed

- LSP compressive residual stresses caused crack closure effect

- The crack closure effect increased minimum load level and reduced load amplitude

- A methodology to consider the LSP residual stresses for the FCP behaviour proposed
\end{abstract}

\begin{abstract}
Laser shock peening (LSP) was performed on aluminium alloy AA2024 with a thickness of 2.0 $\mathrm{mm}$. Microstructural studies using the EBSD technique were performed to quantify the micro-texture changes in the material through LSP. A residual stress analysis was performed using synchrotron radiation and a hole drilling technique. Fatigue crack propagation (FCP) tests were performed to investigate the retardation effect of LSP residual stresses. Loadcrack opening displacement records were analysed to obtain the corrected values of load levels considering the effects of the residual stresses. Specimens with the LSP treatment reveal a significant retardation of the FCP. The presence of compressive residual stresses caused the crack closure effect, which increased the level of minimum load and therefore reduced the effective load amplitude. An original methodology to consider LSP-induced residual stresses on the FCP behaviour was proposed.
\end{abstract}

\section{Keywords}

LSP, AA2024, microstructure, EBSD, fatigue crack propagation, residual stress.

Paper type Research paper

\section{Introduction}

Laser shock peening (LSP) has been successfully used for extending the fatigue life of critical components. LSP imparts residual stresses that are considerably deeper than those of traditional methods, such as sand blasting or shot peening. Several researchers investigated LSP for retarding the growth of long fatigue cracks as well as its effect on mechanical properties (Tan et al., 2004 [15]; Hatamleh et al., 2007 [6]; Hatamleh, 2009 [7]; Hombergsmeier et al., 2014 [8]; Bergant et al. 2016 [1]). Tan et al. (2004) [15] investigated the effects of LSP on the initiation and propagation of fatigue cracks in $2.5 \mathrm{~mm}$-thick AA2024-T3 specimens with various notch geometries. The results clearly indicated that LSP is an effective surface treatment technique for suppressing fatigue crack growth in aluminium alloys with various notch configurations. 
Hatamleh et al. (2007) [6] investigated the influence of LSP on the growth of fatigue cracks in friction-stir-welded sheets of 7075-T7351 aluminium alloy. The authors observed a significant decrease in the fatigue crack propagation (FCP) rates in specimens with LSP treatment versus those of untreated welded and base material (BM) specimens. The fatigue striation spacing for specimens with the LSP treatment was assessed and determined to be small compared to that of the BM specimens. The reduction in the striation spacing indicates a lower FCP rate and could be partially attributed to the deeper compressive residual stresses induced by the LSP.

Furthermore, a significant improvement in the FCP behaviour was demonstrated by Hombergsmeier et al. (2014) [8] in the case of AA2024T3 M(T) specimens with two LSPstripes outside the initial notch. The retardation effect of LSP on the FCP rate was attributed to high compressive residual stresses, which were generated in $2 \mathrm{~mm}$-thick AA2024 sheets. Bergant et al. (2016) [1] observed an inferior FCP behaviour of $10 \mathrm{~mm}$-thick 6082-T651 C(T) specimens with LSP compared to that of BM specimens. The reason was probable in the unoptimised LSP process, which resulted in higher surface roughness without introducing noticeable compressive residual stresses in the investigated material.

There are few studies that investigate the fatigue crack retardation mechanisms acting on a material with LSP residual stresses. Lados and Apelian (2006) [11] published a comprehensive study on how to quantify and consider the effects of residual stresses generated through different heat treatments in Al-Si-Mg cast alloys on the FCP behaviour. The authors described mathematical techniques to consider residual-stress effects on the FCP rate data along with methods for eliminating residual stresses in specimens for the FCP test. Additionally, they reported mechanisms involved in the FCP through residual stress fields. The goal of the current study is to understand the effects of LSP residual stresses on the FCP behaviour of the commonly used aircraft aluminium alloy AA2024 with T351 heat treatment condition. The primary focus was investigating the retardation mechanisms of LSP-induced residual stresses on the FCP.

\section{Experimental methods}

\subsection{Materials and LSP}

A $2.0 \mathrm{~mm}$-thick aluminium alloy 2024 sheet with T351 heat treatment condition without a clad layer was used in this study. This material is generally used in aircraft applications requiring high strength to weight ratios as well as good fatigue resistance. It is an aluminium alloy with copper as the primary alloying element. The investigated material has an engineering ultimate tensile strength of $490 \mathrm{MPa}$, yield strength of $370 \mathrm{MPa}$, and elongation to fracture of $16 \%$. The Vickers hardness of the material is approximately $150 \mathrm{HV} 0.2$ (Enz et al., 2015 [3]).

LSP was performed using a Q-switched Nd:YAG laser operating at $10 \mathrm{~Hz}$ with a wave length of $1064 \mathrm{~nm}$ and a pulse duration of $10 \mathrm{~ns}$. A diffractive optic was used to deliver $3 \mathrm{~J}$ in a square spot of $3 \mathrm{~mm} \times 3 \mathrm{~mm}$ on a specimen surface covered with an aluminium foil. The LSP was applied on the surface of C(T)50 specimens with an initial crack length $a_{0}=10 \mathrm{~mm}$. The treated specimen area was $15 \mathrm{~mm} \times 15 \mathrm{~mm}$. The sequence of LSP spots is depicted in Figure $1(a)$. On one specimen, the LSP treatment was applied 3 times ( $3 x$ overlapping) at the same 
location, and on two specimens, the LSP treatment was applied 5 times (5x overlapping) at the same location. The LSP treatment was applied on two sides of each $C(T) 50$ specimen. The distance before the area of the LSP patterns and the initial crack tip was $1 \mathrm{~mm}$ for specimen LSP $5 x-1$ and $2 \mathrm{~mm}$ for the two specimens LSP $3 x-2$ and LSP $5 x-2$. The specimens investigated in this study are summarised in Table 1.

(a)

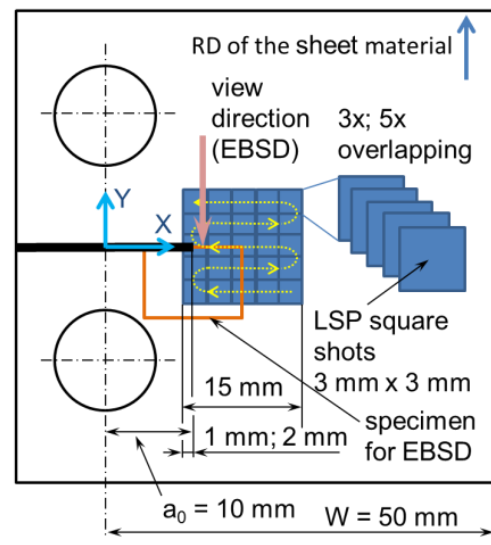

(b)

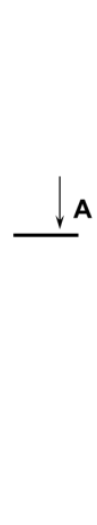

A A

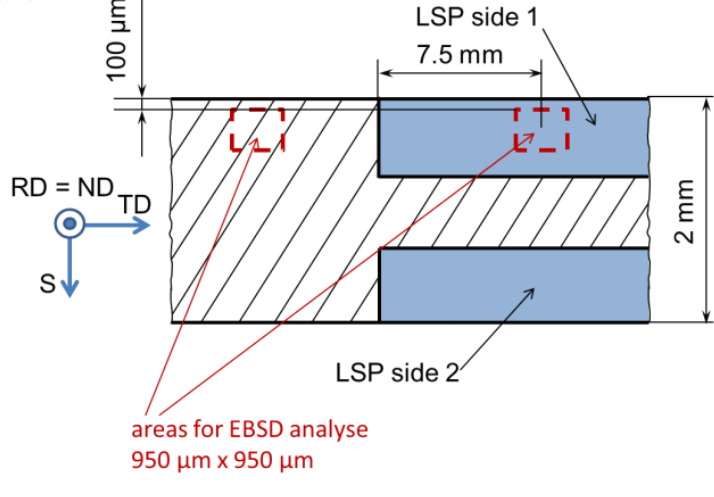

Figure 1. Geometry of the specimens

Notes: (a) Positioning of the LSP patterns on the C(T)50 specimen; and (b) positioning of the areas for the EBSD analysis;

" $R D$ ", "TD" and " $S$ " denote the rolling direction, transverse direction and thickness direction, respectively, of the sheet material. The normal direction (ND) is parallel to the RD of the sheet.

Table I. Specimen documentation

\begin{tabular}{rcc}
\hline $\begin{array}{c}\text { Specimen } \\
\text { identification }\end{array}$ & $\begin{array}{c}\text { Numbers of } \\
\text { LSP } \\
\text { overlapping } \\
\text { areas }\end{array}$ & $\begin{array}{c}\text { Distance of } \\
\text { LSP patterns } \\
\text { before the } \\
\text { crack tip }\end{array}$ \\
\hline BM & - & - \\
LSP $5 x-1$ & 5 & $1 \mathrm{~mm}$ \\
LSP 3x-2 & 3 & $2 \mathrm{~mm}$ \\
LSP $5 x-2$ & 5 & $2 \mathrm{~mm}$ \\
\hline
\end{tabular}

\subsection{Microstructural characterisation}

The microstructure of the BM specimens and the specimens with LSP treatment was studied using SEM with electron back-scatter diffraction (EBSD). The EBSD measurements were performed in the near surface region, where the distance from the specimen surface to the upper corner of the analysed area of $950 \mu \mathrm{m} \times 950 \mu \mathrm{m}$ was approximately $100 \mu \mathrm{m}$. The specimen for the EBSD analysis was extracted from the middle LSP area (Figure 1(b)). The specimen was analysed at $30 \mathrm{kV}$, beam current of $0.3 \mathrm{nA}$, emission current of $75 \mu \mathrm{A}$, magnification of 100x, working distance of $13 \mathrm{~mm}$, step size of $2 \mu \mathrm{m}$, and sample tilt of $70^{\circ}$. The orientation calculation was based on the GSHE method and assumed a triclinic sample symmetry. The data obtained was calculated using the ZAF method. In inverse pole figures, the rolling direction (RD) is [001]. In the orientation, (hkl)[uvw] is the transverse direction parallel to the [uvw] (preferential direction) and (hkl) is parallel to the cross-section surface. The microhardness profiles in the cross-sections of the specimens were obtained using an automated Vickers hardness testing machine. 


\subsection{Residual stress analysis}

The determination of the residual stresses was performed at the P07B station of the HZG beamline HEMS (High Energy Materials Science), which was located in the PETRA III synchrotron radiation source at DESY (Deutsches Elektronen-Synchroton, Germany). The transverse residual stresses $\sigma_{x x}$ and longitudinal residual stresses $\sigma_{y y}$ (Figure 1(a)) were determined in a transmission setup using a photon energy of $87.1 \mathrm{keV}$ (wavelength of $0.1429 \AA$ ) as well as a beam with a cross-section of $0.5 \mathrm{~mm} \times 0.5 \mathrm{~mm}$. A Perkin-Elmer area detector with a pixel size of $200 \mu \mathrm{m}$ at a distance of $1448 \mathrm{~mm}$ from the specimen was used for recording complete diffraction rings. A specimen area of $30 \mathrm{~mm} \times 45 \mathrm{~mm}$ was covered with 30 scan lines of 45 points each, thus resulting in a point mesh with $1 \mathrm{~mm}$ distance between the points and 1426 points (specimen LSP $5 x-2$ ). Only a smaller area of $15 \mathrm{~mm} \times$ $45 \mathrm{~mm}$ was covered for sample LSP $3 \mathrm{x}-2$. Data reduction was performed using the program Fit2d (Hammersley et al., 1996 [5]). The scattering angle $2 \Theta$ of the Al (311) reflection was determined by fitting a Gaussian profile to the measured peaks. Strains were calculated from the shifts in the peak position. The stress-free lattice parameter was determined from the balance of forces along a line from the notch to the opposite specimen edge; the integral of $\sigma_{y y}$ along that line must be zero. The residual stresses were calculated from the strains using a Young's modulus of $E=69.4 \mathrm{GPa}$ and a Poisson's ratio $v=0.35$ for the (311)reflection. For details on the residual stress analysis using diffraction, refer to the book of Lodini and Fitzpatrick (2003) [10].

To determine the depth-resolved residual stresses, the hole drilling equipment "Prism" was used. "Prism" is equipped with an optical electronic speckle pattern interferometer (ESPI) system that provides high-quality full-field data for accurate residual stress determination. This technique has been described in detail by Steinzig and Ponslet (2003) [14].

\subsection{Fatigue crack propagation test}

The specimens used for the FCP test are compact tension specimens based on the ASTM E647-11 standard with a width $W=50 \mathrm{~mm}(\mathrm{C}(\mathrm{T}) 50$ specimens). All of the $\mathrm{C}(\mathrm{T}) 50$ specimens were machined with the loading axis parallel to the rolling direction of the sheet material. An initial crack $a_{0}=10 \mathrm{~mm}$ was introduced by an electro discharge machining. The stress intensity factor range, $\Delta K$, can be calculated based on the mentioned standard as follows:

$$
\Delta K=\frac{\Delta F}{B \sqrt{ } W} \frac{\left(2+\frac{a}{W}\right)\left(0.886+4.64 \frac{a}{W}-13.32\left(\frac{a}{W}\right)^{2}+14.72\left(\frac{a}{W}\right)^{3}-5.6\left(\frac{a}{W}\right)^{4}\right)}{\left(1-\frac{a}{W}\right)^{1.5}},
$$

where $a, B$ and $\Delta F$ are the crack length, specimen thickness $(2 \mathrm{~mm})$ and load alternation, respectively.

The FCP tests were conducted using a servo-hydraulic machine with a capacity of $25 \mathrm{kN}$. Each specimen set was submitted to the same test conditions, i.e., a load ratio, $R=F_{\min } / F_{\max }$ of 0.1 , frequency of $10 \mathrm{~Hz}$ and room temperature. All specimens were polished in the expected area where the crack was supposed to grow to observe the experiment using an optical microscope. The fatigue cracks were monitored on the observable surface using the 
optical microscope. The crack growth was recorded automatically using a clip in the crack mouth, and a second measurement consisted of periodic measurements of the locations of the crack tip using an optical microscope to magnify the crack. For both methods, the crack growth was recorded using the crack length data as a function of the number of cycles. For example, the incremental polynomial method involves fitting a sixth-order polynomial to sets of data points. The method allows the data to be filtered to reduce the margin of error. The crack opening displacement (COD) clip was mounted on the top side of each $C(T) 50$ specimen before the FCP test. During the FCP test, the applied load vs. COD records were measured.

\section{Results}

\subsection{Microstructure and residual stress}

Figure 2 depicts the crystal orientation maps and the corresponding inverse pole figures obtained in the edge region of the AA2024 BM (Figure 2(a) and (b)) and the area influenced by LSP (Figure 2(c) and (d)). The different colours in the crystal orientation maps (Figure 2(a) and (c)) indicate different crystal orientations to the normal direction (ND), i.e., parallel to the crystal direction [001]. The AA2024 BM depicts grains elongated through the rolling process with an average grain size of $36.6 \mu \mathrm{m} \pm 18.8 \mu \mathrm{m}$. From the inverse pole figure (Figure 2(b)), it can be observed that the maximum axial intensity $\left(H_{\max }=1.75 \mathrm{mrd}\right)$ exists at the crystal orientation $<1 \quad 1 \quad 2>/ / N D$ with an aperture angle of approximately $\pm 15^{\circ}$. Furthermore, the maximum transverse in an orientation band occurs in the crystal direction $<012>/ /$ ND with an aperture angle of approximately $\pm 5^{\circ}(H=1.18 \mathrm{mrd})$. Compared to the $\mathrm{BM}$, the area influenced by the LSP depicts axial intensities from $1.74 \mathrm{mrd}$ to $1.93 \mathrm{mrd}$ for the $<112>/ /$ ND and $1.18 \mathrm{mrd}$ to $1.06 \mathrm{mrd}$ for the $<012>/ /$ ND (Figure 2(d)).

The confidence index $(\mathrm{Cl})$ provides a measure of the quality of the EBSD measurement. For the AA2024 BM, $C l=0.55$ indicates the appropriate quality of the EBSD measurement and is comparable to $\mathrm{Cl}=0.54$ of the LSP-influenced area. The differences were obtained in the image quality $(I Q)$ values, i.e., $I Q=3131.61$ for the $B M$ and $I Q=2810.84$ for the LSPinfluenced area. The $I Q$ value is determined using the contrast and brightness of a Kikuchi diffraction image. The Kikuchi diffraction image is used for the identification of the crystal plane (hkl) and determination of the Euler angles $\left(\varphi_{1}, \varphi_{2}, \varphi_{3}\right)$, which is affected by the density of the defects. The high density of defects reduces the brightness and contrast of the Kikuchi diffraction image and thus the IQ-value. Therefore, the lower $1 Q$-value for the LSP influenced area indicates that through the LSP treatment, the density of defects increased. However, there were no modifications observed in the microstructure and grain size in the LSP-influenced area.

The typical (lll 111 ) and ( $\left.\begin{array}{lll}1 & 1 & 0\end{array}\right)$ pole figures of the BM and the LSP-influenced area indicate the presence of a nearly orthorhombic symmetry of the specimens (Figure 3). The position of the pole densities in both the BM and LSP-influenced areas is nearly identical, where the distribution of the orientation bands and pole density is different. The obtained maximum pole densities for the BM $\left(P_{\max }=2.013 \mathrm{mrd}\right)$ and LSP-influenced areas $\left(P_{\max }=2.171 \mathrm{mrd}\right)$ indicate a relatively weak microtexture due to the presence of a high number of different crystal orientations. The pole density in the S-direction of the (llll 111 pole figure obtained from the LSP-influenced area is higher than that of the corresponding BM. Therefore, it can 
be assumed that the interaction between the laser-induced shock waves primarily interacted with crystals in the $<111>/ /$ S crystal direction.

(a)
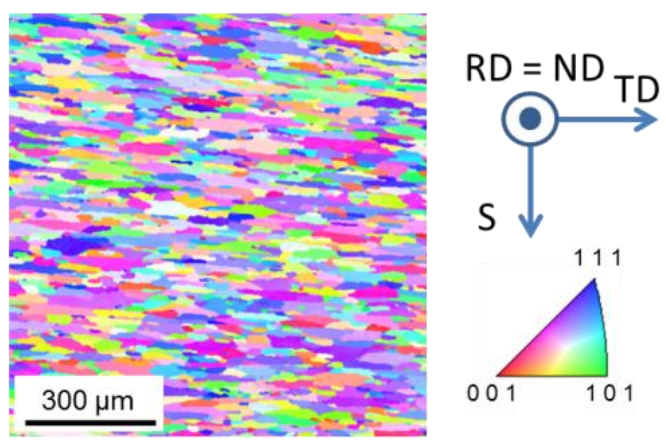

(c)
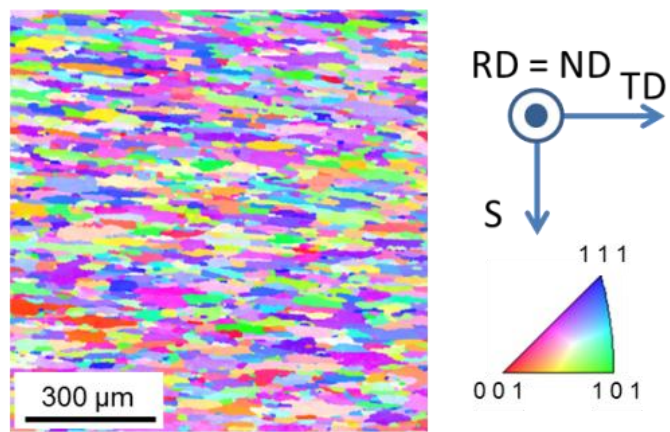

(b)
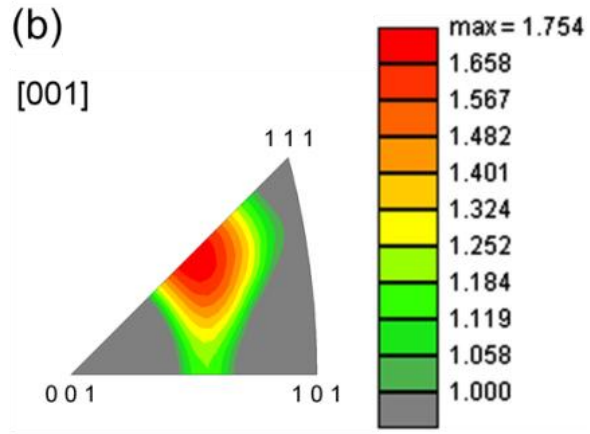

(d)
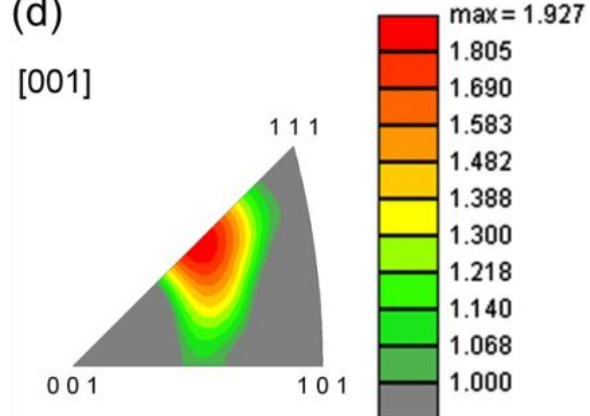

Figure 2. Crystal orientation maps and inverse pole figures obtained from the sub-surface regions of the $B M$ and the $B M$ after the $L S P$ treatment

Notes: $(a, b) B M\left(H_{\max }=1.754 \mathrm{mrd}, L=22, \omega=5^{\circ}\right)$ and $(c, d) B M$ with $L S P\left(H_{\max }=1.927 \mathrm{mrd}\right.$, $\left.L=22, \omega=5^{\circ}\right)$. The distance from the surface to the upper corner of the maps was approximately $100 \mu \mathrm{m}$. ("RD", "TD" and "S" denote the rolling direction, transverse direction and thickness direction, respectively, of the sheet material. The normal direction (ND) is parallel to the RD of the sheet. The LSP treatment was applied with $5 x$ overlapping.

A quantitative EBSD analysis was performed. Details regarding the area-related fractions of the crystal directions are provided in Table 2. It can be clearly seen that all of the represented crystal directions $\langle u v w>/ / N D$ vary throughout the LSP process. The crystallites with crystal directions <1 $12>/ /$ ND and < $12>$ 2 //ND depict the most significant changes in their increased area-related fraction. Furthermore, the area-related fractions of other crystallite directions decreased. Based on these results, it can be assumed that plastic deformations occurred in certain grains without any changes in the structural morphology and grain size.

It can be concluded from the EBSD analysis that the microstructural changes due to local LSP treatment are relatively moderate compared to those obtained in the study by Gariépy et al. (2013) [4]. Gariépy et al. (2013) [4] investigated the microstructural state of AA2024-T351 after it was shot peened using the EBSD analysis. The authors observed an unindexable structure in the first few micrometres with large dislocation densities and extremely small dislocation substructures. They measured significant lattice rotations over distances of approximately $100 \mu \mathrm{m}$ up to a depth of $70 \mu \mathrm{m}$. Contrary to shot peening, the applied LSP treatment in the current study does not depict this type of microstructural damage, even in 
the sub-surface region of the LSP areas. Thus, the LSP is an extremely promising process for the generation of compressive residual stresses without noticeably damaging the specimen surface region. In this study, the selected process parameters for the LSP treatment of AA2024-T3 were suitable to avoid severe surface damages as well as deformations in the microstructure.

(a)

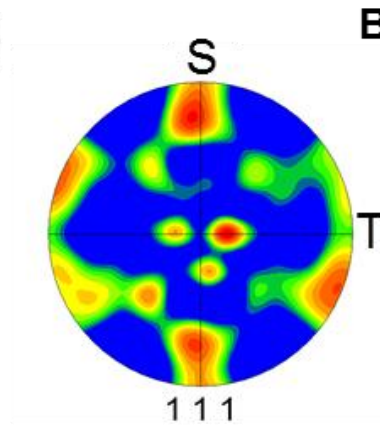

BM

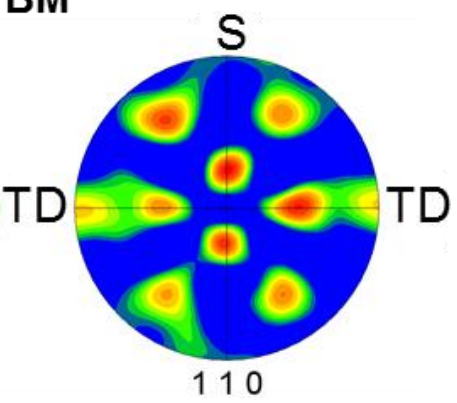

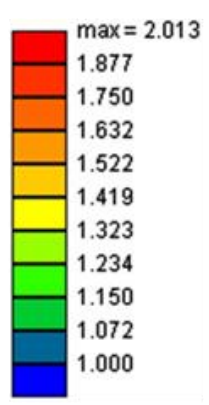

(b)

BM with LSP

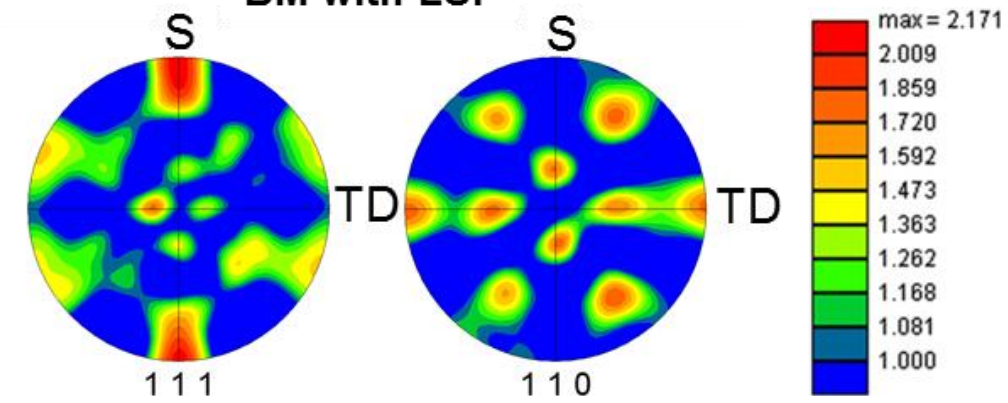

Figure 3. Typical ( $\left.\begin{array}{lll}1 & 1 & 1\end{array}\right)$ and ( $\left.\begin{array}{lll}1 & 1 & 0\end{array}\right)$ pole figures of the sub-surface regions of (a) BM; and (b) BM after the LSP treatment

Notes: (a) BM ( $\left.P_{\max }=2.013 \mathrm{mrd}, L=16, \omega=10^{\circ}\right)$ and $(b) B M$ with $L S P\left(P_{\max }=2.171 \mathrm{mrd}, L=\right.$

$\left.16, \omega=10^{\circ}\right)$. The distance from the surface to the upper corner of the maps was approximately $100 \mu \mathrm{m}$. ("TD" and " $\mathrm{S}$ " denote the transverse direction and the thickness direction, respectively, of the sheet material (Figure 1(b)). The LSP treatment was applied with $5 x$ overlapping.

The results of the residual stress analysis (RSA) using synchrotron radiation are illustrated in Figure 4. It should be noted that the diffraction-based RSA in the transmission setup yields the stresses integrated over the sample thickness. The calculated full width at half maximum (FWHM) maps for the two specimens with LSP treatment indicate a relatively low LSPinduced plasticity in the area of the LSP treatment (indicated by the dashed square) (Figures $4(a)$ and (b)). It is in good accordance with the EBSD results mentioned above, which indicated only rotation of the crystals without any deformation. The low work-hardening is a typical property of the LSP process based on the process conditions and parameters adjusted to AA2024-T351, as indicated in this study. Rouleau et al. (2011) [12] emphasised that the first interesting result is the negligible work-hardening and plastic deformation effects after the LSP treatment of the Al-Cu-Li alloy AA2050-T8. 
Table 2. Area-related fractions of the crystals directions in the BM and LSP-influenced

The measured residual stress maps are illustrated in Figures 4(c)-(f). Close to the crack tip, nearly tensile through-the-thickness residual stresses are present. In the range of a/W values of approximately 0.27 to 0.48 , compressive through-the-thickness residual stresses are present. The values of the compressive residual stresses and sizes of the areas with compressive residual stresses are nearly comparable between the two specimens with the LSP treatment (LSP 3x -2, Figures 4(c) and (e), and LSP 5x -2, Figures $4(\mathrm{~d})$ and (f)). The compressive through-the-thickness residual stresses are compensated with tensile throughthe-thickness residual stresses, which have the highest values at the boundaries of the LSP treated areas. The tensile through-the-thickness residual stresses in the horizontal $(x)$ direction, $\sigma_{x x}$, depict the highest values at the LSP boundaries in the vertical (y)-direction ( $y$ $=-7.5 \mathrm{~mm}$ and $y=7.5 \mathrm{~mm}$, Figures $4(\mathrm{c})$ and (d). In contrast to $\sigma_{x x}$, the tensile trough-thethickness residual stresses in the $y$-direction, $\sigma_{y y}$, depict their highest values at the LSP boundary in the horizontal (crack propagation) direction $(a / W=0.5$, Figures $4(\mathrm{e})$ and (f)).

Figure 5 illustrates the residual stress measurement results obtained using the hole drilling technique. The measurements were performed at 3 locations in the middle of the LSP area from both sides of the specimen. Using a tool diameter of $2.0 \mathrm{~mm}$, it was possible to obtain the residual stress profiles up to a half specimen depth $(1 \mathrm{~mm})$. The measurement results obtained from both sides are provided in Figure 5. The BM specimen depicts a non-uniform depth-resolved residual stress profile, where the residual stresses $\sigma_{y y}$ are slightly higher than the residual stresses in the $x$-direction, $\sigma_{x x}$. The surface regions depict tensile stresses, which are compensated through the compressive residual stresses in the core region of the $\mathrm{BM}$. This residual stress state can be caused by the rolling process of the sheet material.

The specimen with the LSP treatment indicates the highest compressive residual stress at depths of approximately $0.2 \mathrm{~mm}$ (LSP side 1) and $0.1 \mathrm{~mm}$ (from the specimen surface, depth value 1.9 in Figure 5, LSP side 2). The compressive residual stresses, $\sigma_{y y}$, are significantly higher than the compressive residual stresses $\sigma_{x x}$. It should be noted that the obtained values of the compressive residual stresses of approximately $450 \mathrm{MPa}$ are higher than the yield strength of the investigated material $(370 \mathrm{MPa})$. Because the hole drilling technique 
overestimates the residual stress values when they are close to the yield strength of the material, as investigated by Chupakhin et al. (2015) [2], the actual residual stress values should be lower. Nevertheless, the hole drilling technique provides a useable qualitative result. It can be seen that even in the centre of the specimen, the compressive residual stresses occur in both directions. The results indicate that by using the LSP treatment, the generation of through-the-thickness compressive residual stresses is possible.
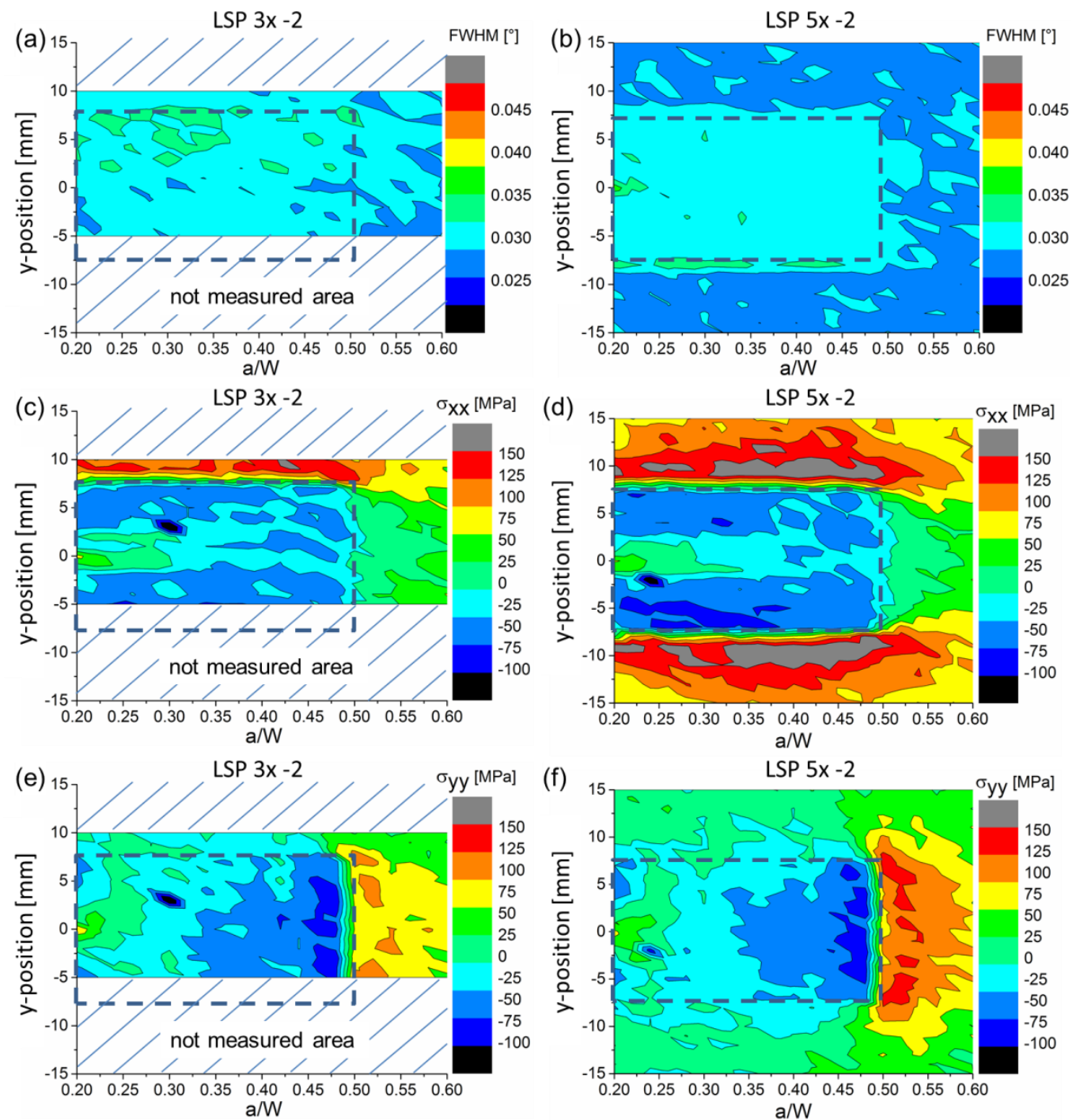

Figure 4. FWHM and RS maps for 2 specimens with LSP treatment

Notes: (a), (c) and (e) obtained for specimen LSP 3x-2 (3x overlapping); and (b), (d) and ( $f$ ) obtained for specimen LSP $5 x-2$ (5x overlapping) 


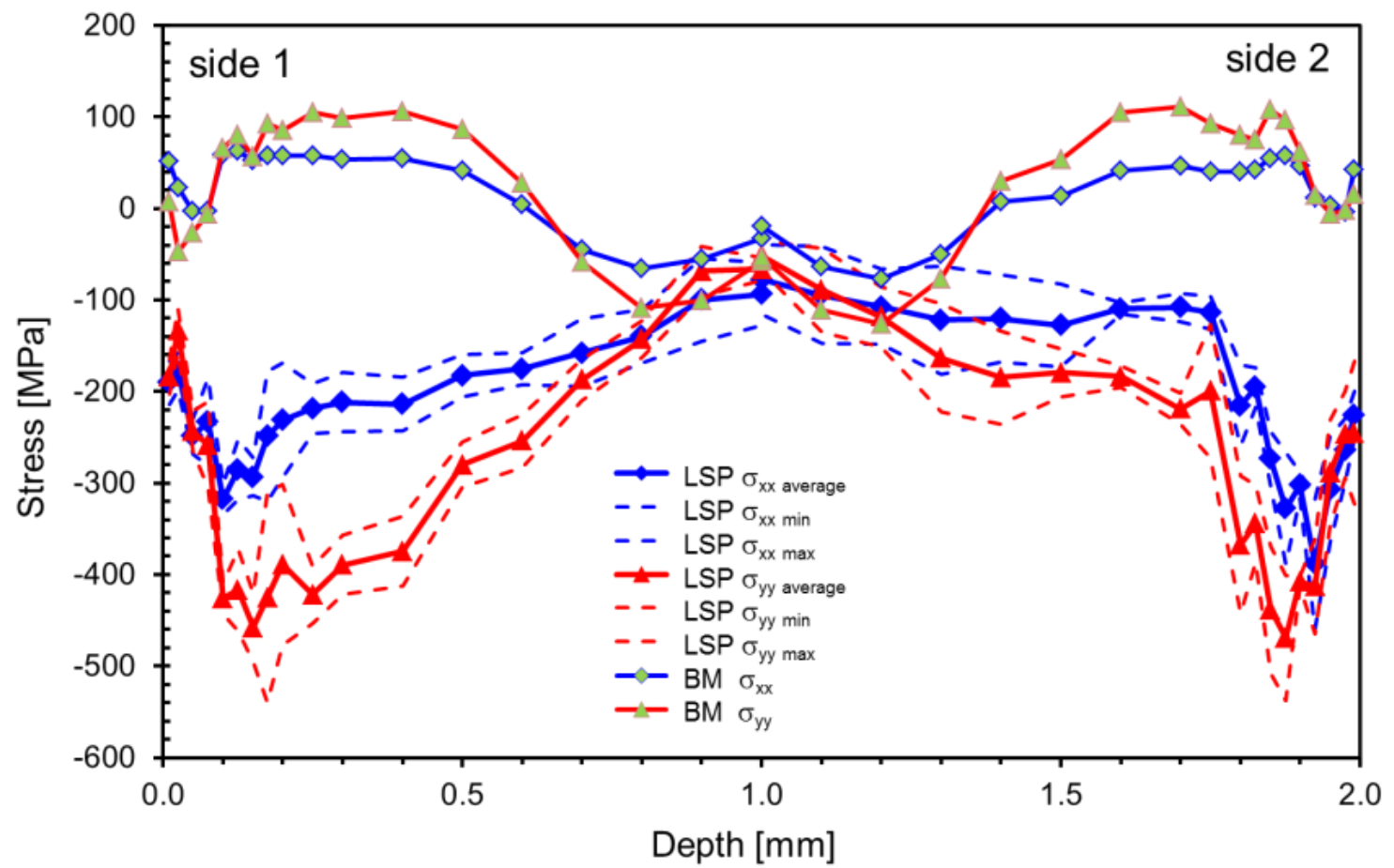

Figure 5. Residual stress profile measured using the hole drilling method for the $C(T) 50 B M$ specimen and the specimen with LSP (5x overlapping) at three points in the middle of the LSP-area with an average curve from both sides of the specimen

The results of the microhardness measurements are depicted in Figure 6 . The microhardness profiles were measured across the specimen depth in a cross-section of a BM specimen and a specimen with LSP treatment. The results indicate that a slight microhardness increase occurs from approximately $150 \mathrm{HV} 0.1$ (mean microhardness of the $\mathrm{BM}$ ) in the surface regions of the specimen with LSP treatment to a microhardness-value of approximately 175 HV 0.1. The microhardness profile of the specimen with the LSP treatment can be correlated with the residual stress profiles illustrated in Figure 5 . For the measured residual stress profiles, the effects of the LSP are more pronounced on side 1 (1st side for the LSP treatment), where the maximum compressive residual stress, $\sigma_{y y}$, is at a depth of approximately $0.2 \mathrm{~mm}$ (from the specimen surface). The maximum compressive residual stress, $\sigma_{y y}$, on side 2 is located at a depth of approximately $0.1 \mathrm{~mm}$ (from the specimen surface, at a depth value of approximately 1.9 in the diagram). The results of the microhardness measurements indicate the same trend as the residual stress measurement results, in which a slight increase in the microhardness is more pronounced on side 1 (Figure $5)$. The increase in the microhardness by around $5 \%$ in areas with highest compressive residual stress is in good agreement with the model proposed by Huber and Heerens (2008) [9], assuming that only the uniaxial compressive residual stresses $\sigma_{x x}$ remains present in the cross-sectioned and indented surface. Because the increase in microhardness can be fully attributed to the effect of residual stress, possible contributions from work hardening due to the LSP treatment are insignificant. 


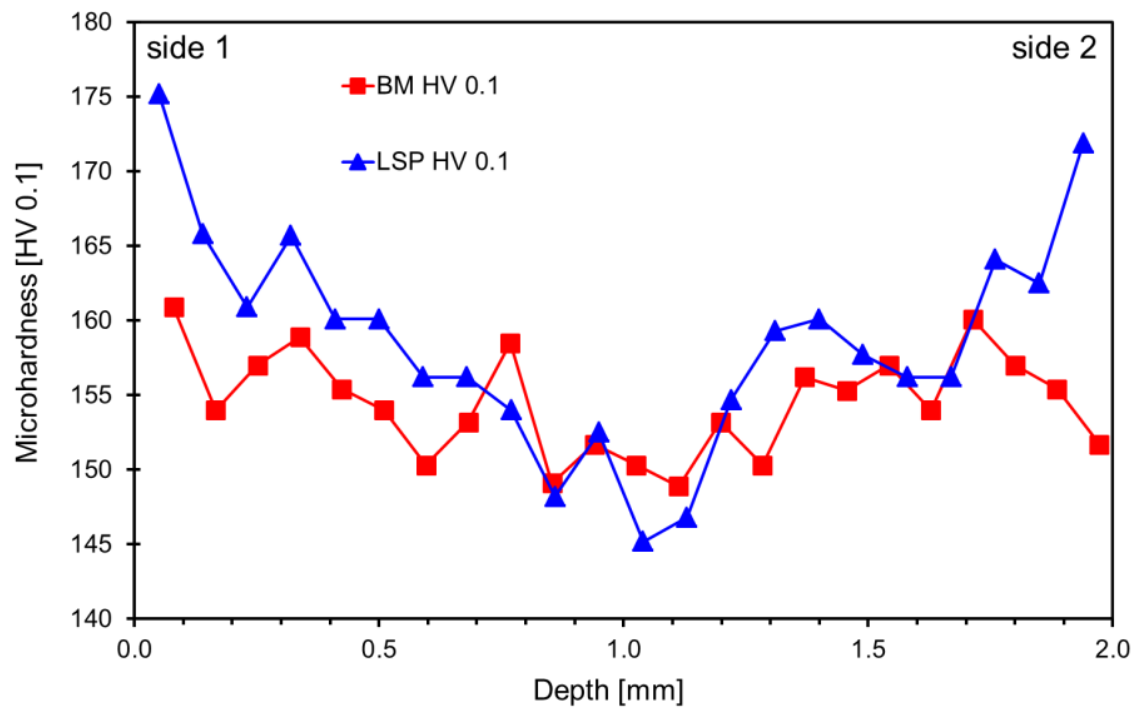

Figure 6. Microhardness profiles of the BM specimen and the BM specimen with LSP treatment (LSP $5 x-1)$

\subsection{Fatigue crack propagation}

Figure 7 depicts the FCP test results. The specimens with the LSP treatment depict a significantly higher number of cycles required for propagating the fatigue crack to an $a / W$ value of 0.6 (crack length, $a$, divided by the $C(T) 50$ specimen width from the pin holes, $W$ (50 $\mathrm{mm}$ ), Figure $7(\mathrm{a})$ ). In the beginning of the FCP test up to approximately 47,000 cycles, specimens LSP $3 x-2$ and LSP $5 x-1$ indicate slightly higher values of $a / W$. This result can be explained due to the presence of tensile through-the-thickness residual stresses in the front of the crack tip, which are depicted in Figure 4(e) for specimen LSP 3x-2. The fatigue life of the specimens with the LSP treatment with $5 x$ overlapping increased by a factor of nearly 3.5 compared to the BM specimen. The FCP rate, $d a / d N$ vs. a/W, as indicated in Figure $7(b)$, demonstrates a significant retardation in the FCP when the crack propagates through the LSP area with high compressive through-the-thickness residual stresses. The retardation of the FCP rate is more significant for specimens with the LSP treatment and $5 x$ overlapping (LSP $5 x-1$ and LSP $5 x-2$ ). At an $a / W$ value of approximately 0.5 (border of the LSP area), where tensile through-the-thickness residual stresses occur, the FCP rate increases with an increasing $a / W$. At an a/W value of approximately 0.6 , the FCP rates of specimens with the LSP treatment reach the FCP rate of the BM specimen.
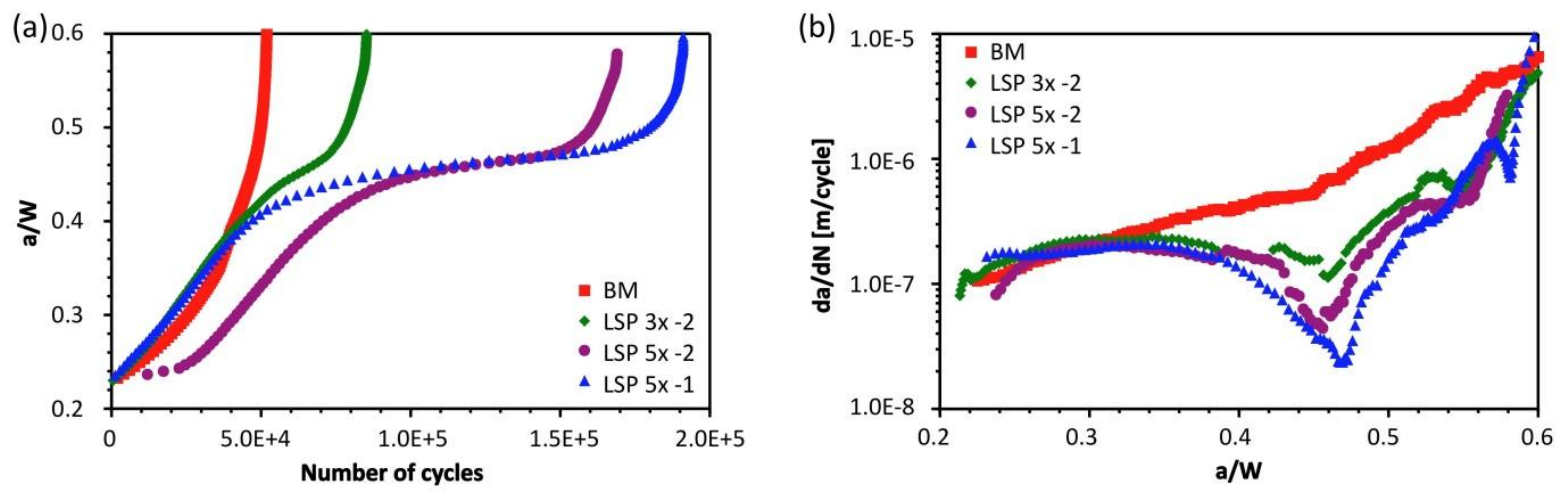

Figure 7. FCP test results

Notes: (a) $a / W$ vs. number of cycles; and (b) da/dN vs. $a / W$ 


\subsection{Fractography}

A fractographical analysis was performed to understand the microstructural issues of the FCP in specimens with higher residual stresses induced through the LSP treatment. The fatigue cracks in specimens with the LSP treatments suffered several crack closures in the region with compressive residual stresses, which changed the crack paths. These effects were observed in all specimens with the LSP treatment. Figure 8 depicts a comparison of the fracture surface for the BM and the specimen with the LSP treatment. At the edges of the specimen with the LSP treatment, plasticised areas with secondary cracks were observed (Figures $8(b)$ and $(f))$. These effects were not observed in the BM specimens, where shear lips are the typical features of the fractured surfaces (Figures 8(a), (c) and (e)). The fractography results indicate that the areas of crack closures were caused by the presence of compressive residual stresses for specimens with the LSP treatment, which are clearly visible by the plasticised surfaces.

\section{Consideration of LSP-induced residual stresses in the evaluation of the FCP test results} To understand the retardation of the FCP in the specimen with the LSP treatment, the hysteretic load vs. the COD records obtained at different crack lengths were analysed. Throughout the analysed $a / W$ region from the beginning of the FCP test $(a / W=0.2)$ to the end of the FCP test $(a / W=0.6)$, the record for the BM specimen is linear and does not indicate any noticeable inflection points (as can be seen at the beginning of the test, at $a / W$ $=0.22 \mathrm{~mm}$, and close to the end of the test, at a $/ \mathrm{W}=0.56$, Figure 9 , red curves). In contrast to the BM specimen, the specimen with the LSP treatment depicts inflection points in the load vs. COD records (blue curves in Figure 9). The records obtained at different $a / W$ values depict different curvatures for the first parts of the curves up to the inflection points. At an $a / W=0.22$, the record of the BM with the LSP indicates a negative curvature (syncline) up to the inflection point at a load value of approximately $0.64 \mathrm{kN}$ (Figure 9(a)). Up to the inflection point, the gradient of the curve is lower than the gradient of the second part of the curve. The gradient of the second part of the curve is comparable to the gradient of the BM curve obtained at the same $a / W$ value. The lower gradient of the first part of the curve indicates that tensile residual stresses occur.

For the load vs. COD record for the specimen with the LSP treatment obtained at the beginning of the test, the $\triangle C O D$ indicated the difference between the upper (linear) part of the curve and the first part of the curve through the tensile residual stress located on the right side of the load vs. COD record (Figure 9(a)). By considering that the $\triangle C O D$ before the inflection point (distance between points $A$ and $D$, Figure 9(a)) resulted from the combination of the effects of the tensile residual stresses, $\triangle C O D_{\text {tensile Rs, }}$ and the applied load, $\triangle C O D_{\text {apl load, }}$ the $\triangle C O D_{\text {corr }}$ that should contribute to the increase in the applied load due to the presence of tensile stresses as the distance between points $C$ and $D$ can be estimated as $\triangle C O D_{\text {apl load }}-\triangle C O D_{\text {tensile RS }}$ (distance between points $\mathrm{A}$ and $\mathrm{B}$ is equal to the distance between points $\mathrm{B}$ and $\mathrm{C}$ ). The assumption is that for the $\triangle C O D$ before the inflection point, a part from the applied load for $\triangle C O D_{\text {corr }}$ is not considered in the first part of the record, i.e., before the inflection point, and therefore has to be added to the end linear part of the record. The virtual corrected maximal load, $F_{\max c o r r}$, which considers the effects of the tensile stresses, should include the linear part of the increase to point $E$ to incorporate $\triangle C O D_{\text {corr }}$ (distance between points $\mathrm{C}$ and $\mathrm{D}$ is equal to the distance between points $\mathrm{C}^{\prime}$ and 
$\left.D^{\prime}\right)$. The resulting $F_{\max c o r r}$ is approximately $1.4 \mathrm{kN}$ for the load vs. COD record obtained at an $a / W$ value of 0.22 , as indicated in Figure $9(\mathrm{a})$.

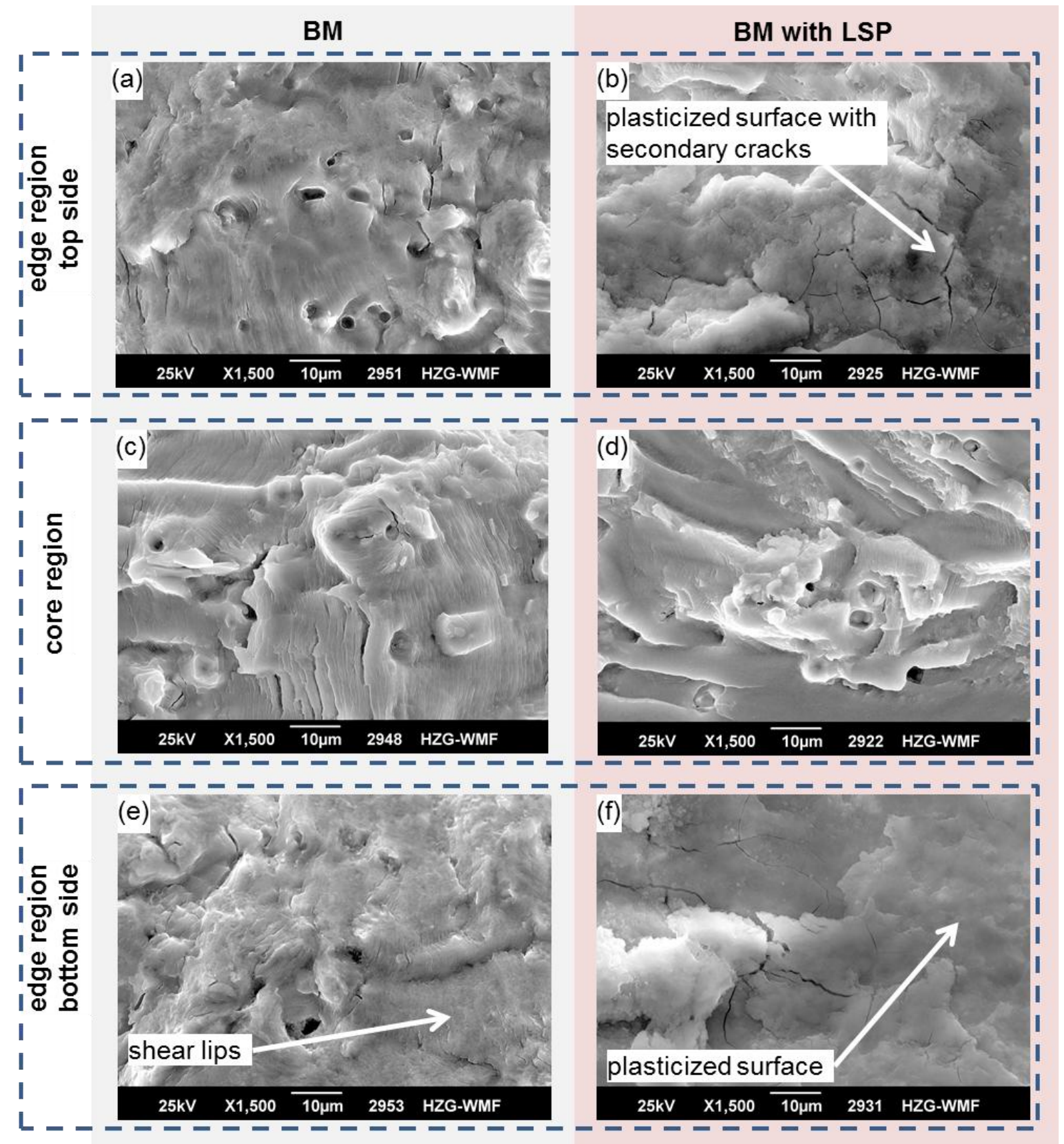

Figure 8. Overview of the fractured surfaces

Notes: (a), (c) and (e) for the BM specimens; and

(b), (d) and (f) for the specimens with the LSP treatment (LSP 5x-1, Table 1)

The load vs. COD record obtained at $a / W=0.56$ initially depicts a positive curvature (anticline). The higher gradient of the first part of the curve indicates that the compressive residual stresses were present and caused the crack closure that influenced the minimum load, $F_{\text {min }}$, which increased up to the inflection point of the hysteretic curve. Furthermore, the inflection point of the record can be considered as the opening load. The $\triangle C O D$ indicated the difference between the upper (linear) part of the curve and the first part of the curve through the compressive residual stress located on the left side of the load vs. 
COD record. By considering the crack closure effects, the corrected minimal load, $F_{\text {min }}$ corr should be increased to the inflection point of the load vs. COD record (approximately 0.52 kN, Figure $9(b))$.
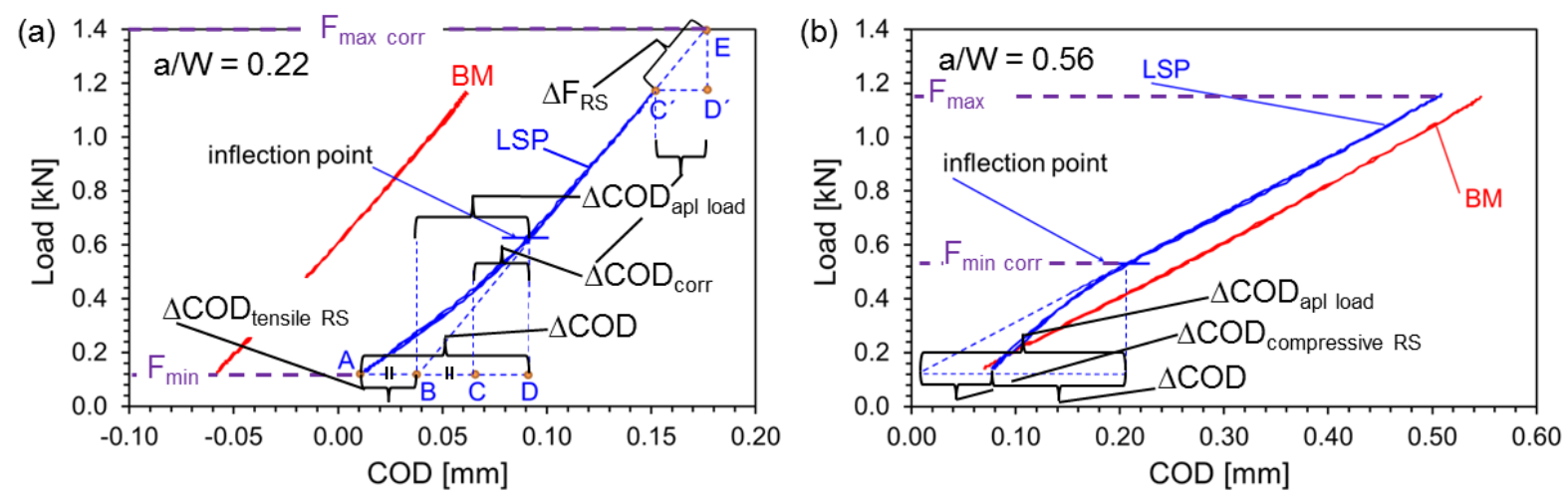

Figure 9. Load vs. COD records obtained at different $a / W$ values

Notes: (a) BM specimen and BM specimen with the LSP treatment (LSP 5x-1, Table 1) in the beginning of the FCP test; and (b) BM specimen and BM specimen with the LSP treatment (LSP $5 x-1$, Table 1) at an a/W value of 0.56

Based on the considerations described above, the load vs. COD records for the BM specimen and the specimens with the LSP treatment were analysed, and $F_{\text {max corr }}$ and $F_{\text {min corr }}$ were calculated for the tensile residual stress and compressive residual stress regions, respectively. Then, the effective load ratio, $R_{\text {eff, }}$ was calculated considering the corrected load levels. The results are presented in Figure 10(a). The crack closure from the compressive residual stress for specimens with the LSP treatment affected the R-ratio (increased the level of minimum load) and therefore reduced the effective load amplitude in the areas with compressive residual stresses. In the tensile residual stress regions, the $R$ ratio was insignificantly reduced. The $R$-ratio for the $\mathrm{BM}$ specimen remained nearly constant during the FCP test. A similar retardation mechanism has been described also for FCP in laser heating induced residual stresses by Schnubel and Huber (2012) [13], with the difference, that there tensile residual stresses are induced in the treated area, surrounded by compressive residual stresses.

The FCP rates vs. stress intensity factor range, $\Delta K$, are depicted in Figure $10(\mathrm{~b})$. The $\mathrm{BM}$ specimens depict a typical linear behaviour in the logarithmic coordinates. The calculated $\Delta K$ values for the specimens with the LSP treatment considering the applied loads depict minimum $d a / d N$ values at a $\Delta K$ value of approximately $20 \mathrm{MPaVm}$. By considering the corrected values of $F_{\min }$ and $F_{\max }$ for the calculation of the stress intensity factor range, i.e., the so-called effective stress intensity factor range, $\Delta K_{\text {eff, }}$ the $d a / d N$ vs. $\Delta K_{\text {eff }}$ curves for the specimens with the LSP treatment are close to the curve of the BM specimen. It is evident that the applied corrections for calculating $\Delta K_{\text {eff }}$ are reasonable and consider the effects of the tensile/compressive residual stresses induced by the LSP treatment. 

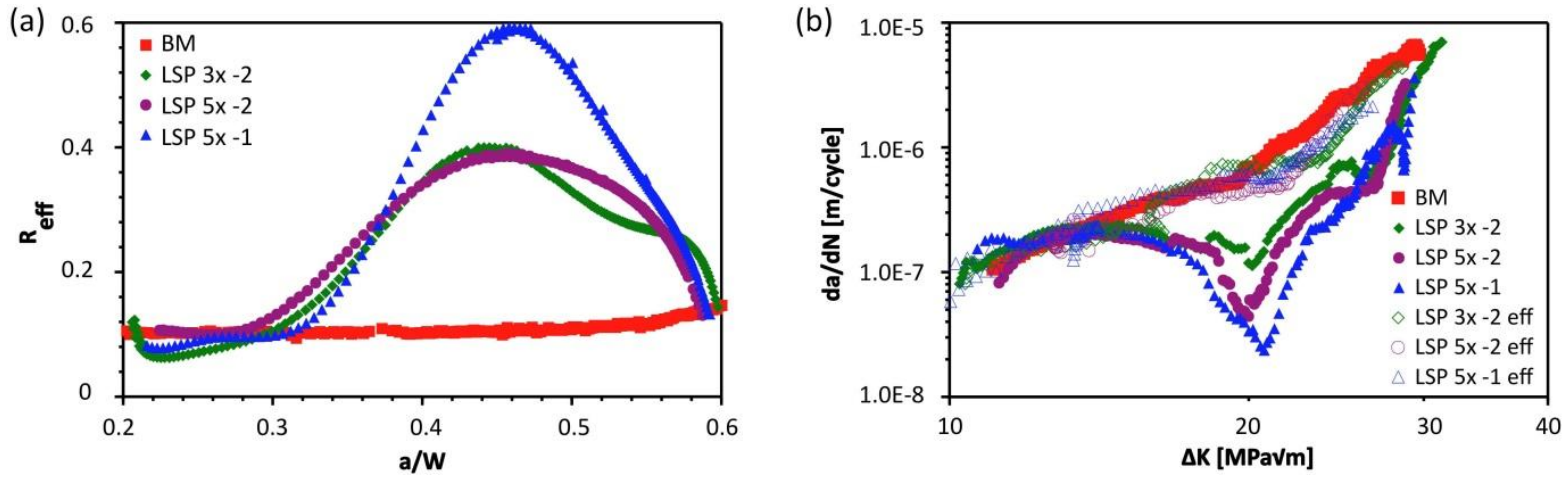

Figure 10. Correction of effective values under consideration of the LSP-induced residual stresses

Notes: (a) Reff vs. $a / W$; and (b) $d a / d N$ vs. $\Delta K$

\section{Conclusions}

- LSP is an effective process for introducing high and deep compressive residual stresses without severe surface damages as well as deformations in the microstructure of thin AA2024 sheets.

- A significant retardation of the FCP through the presence of compressive residual stresses induced by the LSP treatment was observed.

- A crack closure effect in the FCP tests was observed in specimens with the LSP treatment, which is caused by the presence of high compressive residual stresses.

- A methodology to consider the presence of residual stresses for the FCP behaviour of specimens with the LSP treatment was proposed.

- LSP can be considered as a potential tool for the surface treatment of Al-structures in which an NDT inspection can detect cracks. The effects of the LSP treatment are the arrest of cracks and deceleration of the FCP.

\section{Acknowledgements}

We acknowledge Deutsches Elektronen-Synchroton (DESY), Germany for using synchrotron radiation diffraction at the beamline P07B, which is operated by the German Engineering Materials Science Centre (GEMS) of the Helmholtz-Zentrum Geesthacht. Additionally, the study was supported by the research platform ACE of the Materials Mechanics Division of the Helmholtz-Zentrum Geeesthacht. The authors would also like to thank the following project members for their valuable work and support: H. Tek (mechanical testing), L. Moura (using the hole drilling system) and F. Dorn (specimen preparation).

\section{References}

Bergant, Z., Trdan, U., Grum, J. (2016), "Effects of laser shock processing on high cycle fatigue crack growth rate and fracture toughness of aluminium alloy 6082T651", International Journal of Fatigue, Vol. 87, pp. 444-455. behaviour on determination of residual stress profiles using the hole drilling method", The Journal of Strain Analysis for Engineering Design, in press. 
[3] Enz, J., Khomenko, V., Riekehr, S., Ventzke, V., Huber, N., Kashaev, N. (2015), "Single-sided laser beam welding of a dissimilar AA2024-AA7050 T-joint", Materials and Design, Vol. 76, pp. 110-116.

[4] Gariépy, A., Bridier, F., Hoseini, M., Bocher, P., Perron, C., Lévesque, M. (2013), "Experimental and numerical investigation of material heterogeneity in shot peened aluminium alloy AA2024-T351", Surface and Coatings Technology, Vol. 219, pp. 15-30.

[5] Hammersley, A.P., Svensson, S.O., Hanfland, M., Fitch, A.N., Häusermann, D. (1996), "Two-dimensional detector software: From real detector to idealised image or two-theta scan", High Pressure Research, Vol. 14, pp. 235-248.

[6] Hatamleh, O., Lyons, J. and Forman, R. (2007), "Laser and shot peening effects on fatigue crack growth in friction stir welded 7075-T7351 aluminium alloy joints", International Journal of Fatigue, Vol. 29 No. 3, pp. 421-434.

[7] Hatamleh, O. (2009), "A comprehensive investigation on the effects of laser and shot peening on fatigue crack growth in friction stir welded AA2195 joint", International Journal of Fatigue, Vol. 31 No. 5, pp. 974-988.

[8] Hombergsmeier, E., Holzinger, V., Heckenberger, U.C. (2014), "Fatigue crack retardation in LSP and SP treated aluminium specimens", Advanced Materials Research, Vol. 891-892, pp. 986-991.

[9] Huber, N. and Heerens, J. (2008), "On the effect of a general residual stress state on indentation and hardness testing", Acta Materialia, Vol. 56, pp. 6205-6213.

[10] Lodini, A., Fitzpatrick, M.E. (Eds.), (2003), Analysis of residual stress by diffraction using neutron and synchrotron radiation, Taylor \& Francis, London.

[11] Lados, D.A. and Apelian, D. (2006), "The effect of residual stress on the fatigue crack growth behaviour of Al-Si-Mg cast alloys - Mechanisms and corrective mathematical models", Metallurgical and Materials Transactions A, Vol. 37A, pp. 133-145.

[12] Rouleau, B., Peyre, P., Breuils, J., Pelletier, H., Baudin, T., Brisset, F. (2011), "Characterization at a local scale of a laser-shock peened aluminium alloy surface", Applied Surface Science, Vol. 257, pp. 7195-7203.

[13] Schnubel, D. and Huber, N. (2012), "The influence of crack face contact on the prediction of fatigue crack propagation in residual stress fields", Engineering Fracture Mechanics, Vol. 84, pp. 15-24.

[14] Steinzig, M. and Ponslet, E. (2003), "Residual stress measurement using the hole drilling method and laser speckle interferometry: Part 1", Experimental Techniques, Vol. 27 Issue 3, pp. 43-46. 
[15] Tan, Y., Wu, G., Yang, J.M., and Pan, T. (2004), "LSP on fatigue crack growth behaviour of aluminium alloy", Fatigue \& Fracture of Engineering Materials \& Structures, Vol. 27 No. 8, pp. 649-656. 